\title{
Midrange periodicities in sunspot numbers and flare index during solar cycle 23
}

\begin{abstract}
H. Kiliç
Akdeniz University, Faculty of Science and Art, Physics Dept., 07058 Antalya, Turkey

e-mail: hkilic@akdeniz.edu.tr

Received 9 August 2007 / Accepted 21 November 2007

ABSTRACT

Aims. In the present study, the midrange periodicities are investigated in the data of sunspot numbers and solar flare index during solar cycle 23 for a time interval from August 23, 1997 to December 31, 2005.

Methods. For spectral analysis of these data sets, the Date Compensated Discrete Fourier Transform (DCDFT) is used and this technique is applied separately for the data of total disk, northern and southern hemispheres of the Sun.

Results. The power spectrums of the sunspot numbers exhibit several important midrange periodicities at 84.4, 113.7, 133.6 and 220.0 days which are consistent with the findings in previous studies made by several authors. Two peaks at 113.7 and 133.6 days detected from the whole disk data are contributed mainly by the southern and northern hemisphere, respectively. However, the results of this study show that the discrepancies in the periodic variations of the northern and southern hemispheres of the Sun display a kind of asymmetrical behavior. On the other hand, the flare index data covering the same time interval does not demonstrate any significant periodicity. This study shows that the DCDFT technique for the periodic analysis of solar time series is fairly useful.
\end{abstract}

Key words. Sun: activity - sunspots - Sun: flares

\section{Introduction}

Sunspots, the basic indicators of solar activity, show temporal variations from a few days to several years. Periodicities between 27 days and 11 years are called the "midrange" (Bai 2003). Many authors have searched for the existence of midrange periodicities in various solar activity indicators (Bai 2003) following the discovery of a 154-day periodicity (Rieger et al. 1984) during high-energy flares in solar cycle 21 . In the same solar cycle, the near 160-day Rieger type periodicities were also found in flare-related data (Bogart \& Bai 1985; Özgüç \& Ataç 1989; Bai \& Cliver 1990; Kile \& Cliver 1991; Bai 2003; Ballester et al. 2004; Joshi \& Joshi 2005). During solar cycles 19-22, several researchers also looked for midrange periodicities except for 154-day in flare data. While Bai (1987) reported a 51-day periodicity from analysis in the occurrence rate of major flares in solar cycle 19, the periodicities of 78 days (Bogart \& Bai 1985), 84 days and 127 days (Bai \& Sturrock 1991) were obtained by analysis of solar flare data of cycle 20 . During solar cycle 22, it was seen that significant peaks at 74 , 77 and 83 days were present in flare data (Bai 1992; Özgüç \& Ataç 1994; Joshi \& Joshi 2005). Recently, Ataç \& Özgüç (2006) found that the periods at 64,83 and 125 days are in operation during the maximum phase of the solar cycle 23 .

The presence of variations similar to 154-day in the sunspot data during different solar cycle were investigated by several researchers (Lean \& Brueckner 1989; Lean 1990; Carbonell \& Ballester 1990, 1992; Ballester et al. 1999; Krivova \& Solanki 2002; Richardson \& Cane 2005). Oliver et al. (1998) showed that there was a time-frequency coincidence between the occurrence of the periodicity in both sunspot areas and high-energy flares in solar cycle 21. Ballester et al. (2002) confirmed during solar cycle 21 that the periodicity appeared in the photospheric magnetic flux linked to strong magnetic fields. In early solar cycle 23, Richardson \& Cane (2005) found an intermittent quasiperiodicity similar to 150 -day. On the other hand, results of periodicity analysis of sunspot numbers generally agree with the results of sunspot-area analysis (Lean \& Brueckner 1989; Joshi et al. 2006). The analysis of sunspot areas in solar cycles 12-21 showed that the periodicities in the range of 130 to 185 days were present only during epochs of maximum activity and that it appeared intermittently for the interval of 1 to 3 years (Lean 1990). Several authors (Kile \& Cliver 1991; Bai 1992; Oliver \& Ballester 1995) have however reported that, in solar activity indicators during solar cycle 22, there is no evidence for a 154-day periodicity.

Since many authors have applied different methods to a range of sunspot and flare-index data sets, we here reanalyze the periodicity of cycle 23 using a new method for the spectral analysis of solar time series. We analyze the midrange periodicities of sunspot numbers and the solar-flare index, and investigate the causal relationship between periodic behaviors. We use the Date Compensated Discrete Fourier Transform (DCDFT) technique that is an ideal tool for periodicity analysis. Periodic variations are studied individually for the northern and southern hemispheres of the Sun.

\section{Data and analysis}

We use the daily values of sunspot numbers of the Sun provided by the Brussels World Data Center for Sunspot Numbers (SIDC) ${ }^{1}$. The data sets cover from August 23, 1997 to December 31, 2005 which contain the ascending and descending branch of solar cycle 23. Flare index data analyzed for the same

${ }^{1}$ http://sidc.oma.be/DATA/DAILYSSN 
time intervals were taken by the Kandilli Observatory ${ }^{2}$ and by National Geophysical Data Center (NGDC) $)^{3}$. For the periodic analysis of sunspot numbers and flare index data, the data sets of the total disk, the northern and southern hemisphere were analyzed separately. The time span of the data series is 3053 days.

Astronomers very commonly use a modified periodogram (Scargle 1982) to estimate periods occuring in solar indices, such as the sunspot activity and flare index. The periodogram, similar to the method of least squares, is useful for unevenly-sampled data and is often used in tests of the significance of periods in data. For the analysis of data sets considered in this study, however, the Date Compensated Discrete Fourier Transform (DCDFT) first derived by Ferraz-Mello (1981) was used. The method, a lesser known but powerful technique for periodic analysis of time series, was modified and implemented as the CLEANest algorithm by Foster (1995) specifically to analyze the long-term visual data of variable stars from International Databases such as AAVSO (Templeton 2004). The two methods are similar to each other and give equivalent results in many cases, if the data gaps do not have nearly the same period as the signal itself (Foster 1995). However, the DCDFT method has not been used much for solar data. It is applied here for the first time to a solar-type data series.

As the sunspot or flare data are not statistically independent but correlated, the power $P_{N}$ follows an exponential distribution (Horne \& Baliunas 1986); i.e., the probability of the power density at a given frequency being greater than $k$ by chance is given by

$P_{r}\left[P_{N}\left(w_{0}\right)>z\right]=\exp (-z / k)$,

where the normalization factor $k$, which is due to event correlation, should be determined empirically (Delache et al. 1985; Bai \& Cliver 1990).

Since the DCDFT uses an algorithm that enables us to compute a periodogram in any range of frequency (or period), we scanned the power spectra of total disk, northern and southern hemispheres of the Sun for periods in the range 50-250 days. The result is that the power spectrum calculated for daily sunspot numbers of the full solar disk showed the highest peak at 113.7 days with height 22.57 (Fig. 2). We then studied the cumulative power distribution, shown in Fig. 1, derived using the method described above. It is seen from this figure that for all 147 frequencies the power exceeds zero. At only one frequency $(0.0121 / \mathrm{d})$ the power was at its maximum value, 22.57. For lower values of power, the distribution can be well fitted by the equation which is shown in Fig. 1, as expected from Eq. (1). Thus, the normalized power spectrum exhibited in Fig. 2 is found by dividing the powers by 2.48 . For all spectra, the same procedure for normalization was followed. According to Scargle (1982) and Horne \& Baliunas (1986), the False alarm probability (FAP) that estimates the statistical significance of a peak in the power spectrum is given by

$F=1-\left[1-\exp \left(-Z_{m}\right)\right]^{N}$,

where $Z_{m}$ is the height of the peak in the normalized power spectrum and $N$ is the number of independent frequencies (nif). Fourier components computed for frequencies at intervals of the independent Fourier spacing, $\Delta f_{\text {nif }}=\tau^{-1}$, where $\tau$ is the time span of the data, are totally independent (Scargle 1982).

\footnotetext{
2 ftp://ftp.koeri.boun. edu.tr/pub/astronomy/ flare_index

3 ftp://ftp.ngdc.noaa.gov/stp/solar_data/solar_flares/ index
}

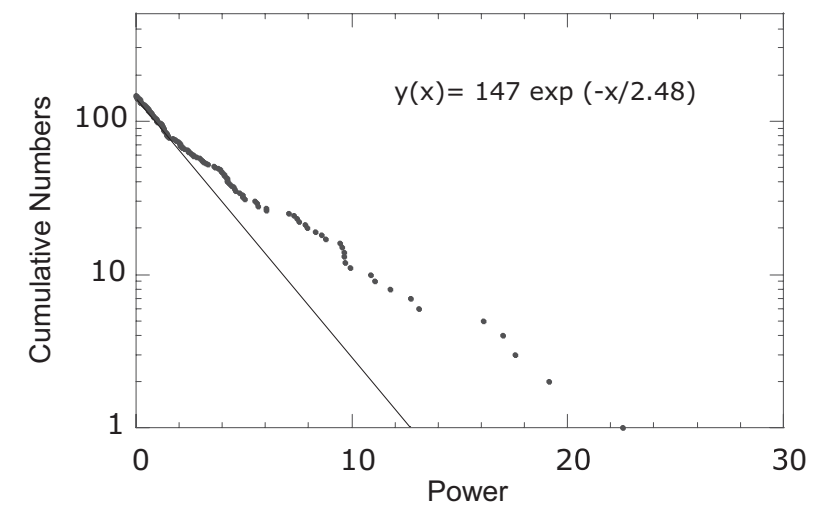

Fig. 1. DCDFT power distribution of daily values of sunspot numbers of total disk for the time interval from August 23, 1997 to December 31, 2005. The vertical axis is the number of frequencies for which power exceeds $x$. The straight line shows the fit to the points for lower values of power.

However, de Jarger (1987) has shown by Monte-Carlo simulations that the Fourier powers taken at intervals of one-third of the independent Fourier spacing are still statistically independent. Here, $\tau$ is 3053 days for all data sets and the number of independent frequencies $N$ in the search window from frequency $f_{1}$ to $f_{2}$ is calculated to be $f_{2}-f_{1} / f_{\text {nif }}$ or $\left(f_{2}-f_{1}\right) \tau$. For $f_{1}=0.004 /$ day (250 days) and $f_{2}=0.02 /$ day (50 days), the number of independent frequencies of all data sets is 49 (according to de Jarger 1987, 147 independent frequencies). We derive a power spectrum of normalized peak amplitude of 9.1 at 113.7 days, by oversampling, by considering the peak height number of frequencies for the total disk in Fig. 2. The oversampling enables the peak value to be estimated more accurately. If $Z_{m}=9.1$ and $N=147$ are substituted in Eq. (2), the FAP $F=0.016$ is obtained; i.e., the probability of finding such a high peak by chance is about $1.6 \%$. To estimate the statistical significance of strong peaks in the power spectra, the same analysis has been applied to another time series.

Since peaks in a spectrum may appear from aliasing, the FAP criterion, alone, is insufficient for evaluating whether or not a strong peak in a spectrum is a real periodicity in the time series (Lean \& Brueckner 1989). One useful method of evaluating this is to subtract the sine curves of their periods from the original time series and then to recompute the periodogram (Horne \& Baliunas 1986). A good computational method for this procedure is provided by Ferraz-Mello (1981). After subtraction of the sine curves, the normalized power spectra demonstrate how the peaks are removed from the power spectra of time-series (Fig. 3).

\section{Discussion and conclusions}

This paper presents the results of the periodic analysis of the sunspots and solar flare index during solar cycle 23 . The spectral analysis of daily sunspot numbers in solar cycle 23 reveals the presence of significant periodicities. However, the power spectrum of daily flare index for the same interval does not show any evident peak with FAP value below 50\% (Fig. 4). Table 1 summarizes the meaningful periodicities detected in these data series.

The power spectrum of sunspot numbers for the total surface of the Sun in the bottom panel of Fig. 2 demonstrates two important peaks at 113.7 days and 133.6 days, which are detected in the southern and the northern hemisphere of the Sun, respectively. While another peak in the northern hemisphere appears at 

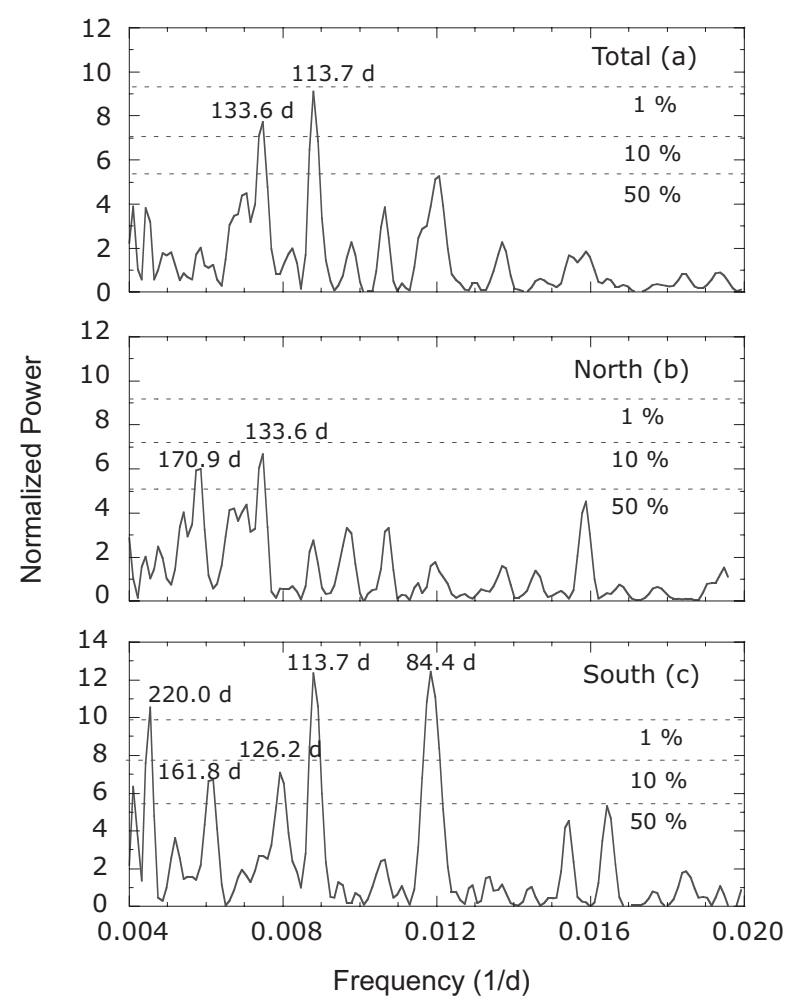

Fig. 2. Normalized power spectrum of the daily sunspot numbers for the total disk a), northern b) and southern c) hemisphere of the Sun for the time interval from August 23, 1997 to December 31, 2005. Power spectra are computed for the period interval of 50-250 days. The dotted lines in each panels show the FAP significance levels.

170.9 days, the southern part of the solar disk shows significant peaks at 84.4 and 220.0 days (Figs. 2b,c).

The most prominent peaks at 84.4, 113.7 and 133.6 days found in the present analysis are in agreement with the results of Joshi et al. (2006). In addition, the periodicities of 133.6 and the 170.9 days agree with the results of Lean (1990) and Richardson $\&$ Cane (2005). Bai (2003) found a periodicity of 129 days in the solar flare occurrence of cycle 23 and suggested that this upheld the findings of Bai \& Sturrock (1991) that 25.5 days is a fundamental period of the Sun. In the current cycle, while Joshi \& Joshi (2005) obtained a peak at 123 days from an analysis of X-ray flare index, Ataç \& Özgüç (2006) detected a 125.9-day periodicity in $\mathrm{H}-\alpha$ flare index. These periodicities are close to the peak at 126.2 days detected in the present analysis. The periodicity of 83 days in the study of Ataç \& Özgüç mentioned above is also close to 84.4-day periodicity detected in this work. During solar cycle 22, Oliver \& Ballester (1995) analyzed the sunspot areas and they showed that a periodicity close to 86 days was statistically significant. A similar periodicity was detected in energetic X-ray flares by Landscheidt (1986) during solar cycle 20 and 21, and in major solar flares by Bai (1992) during cycle 20. The other peak at 161.8 days in the power spectrum of southern hemisphere (Fig. 2c bottom panel) is very close to the periodicity of around 160 days in the photospheric magnetic flux analyzed by Ballester et al. $(2002,2004)$ during solar cycle 21-23. In the light of their analysis, they proposed that the discrepancy of periodic emergence between cycles 21 and 23 can appear in two ways: for cycle 21 , the periodic emergence of magnetic flux occurred mainly within already-developed sunspot groups. This gave rise to an enhancement in their magnetic complexity, which triggered periodic energetic flares. During cycle 23, the periodic
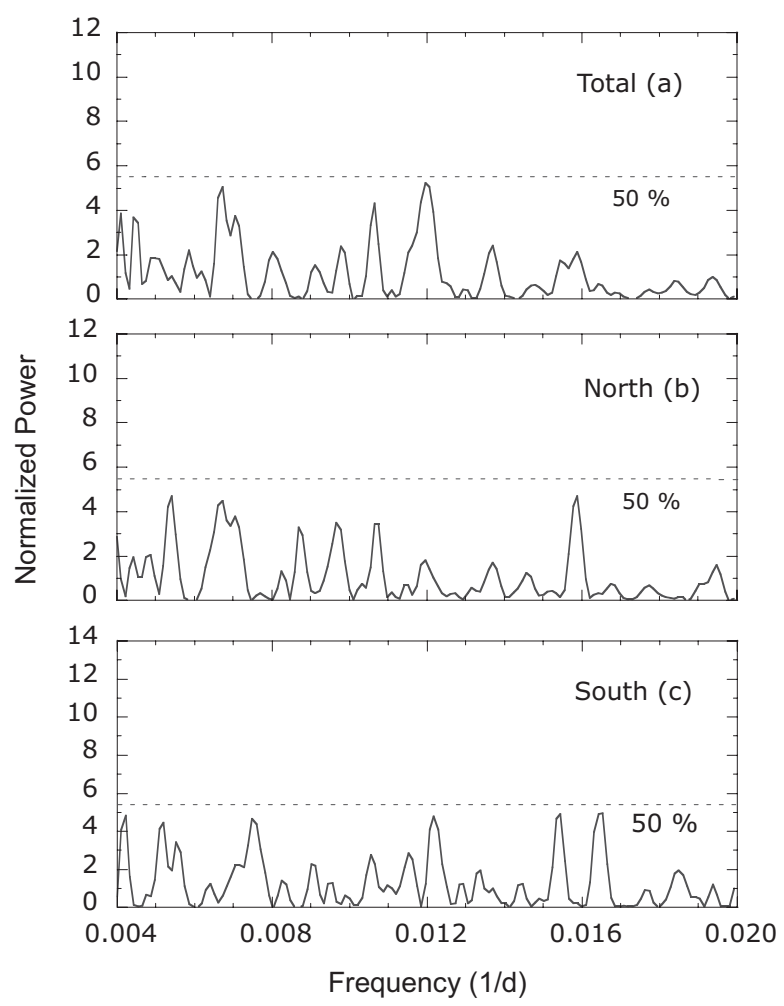

Fig. 3. Same as Fig. 2, but normalized power spectra obtained after subtraction of the sine curves of significant peaks for the total disk a), northern b) and southern c) hemisphere of the Sun for the time interval from August 23, 1997 to December 31, 2005.

Table 1. Periodicities (in day) found by spectral analysis of sunspot numbers in the total solar disk, northern and southern hemispheres of the Sun and False alarm probability (FAP) values (\%) in brackets.

\begin{tabular}{ccc}
\hline \hline Total & North & South \\
\hline$\ldots$ & $\ldots$ & $84.4(0.06)$ \\
$113.7(6.0)$ & $\ldots$ & $113.7(0.06)$ \\
$133.6(1.6)$ & $133.6(17.0)$ & $126.2(11.0)$ \\
$\ldots$ & $170.9(30.0)$ & $161.8(16.0)$ \\
$\ldots$ & $\ldots$ & $220.0(0.4)$ \\
\hline
\end{tabular}

emergence of magnetic flux has shown a mixed behavior, partly occurring away from already-developed sunspot groups. For cycles 18-23, Kane (2005) completed an extensive analysis of several solar indices and detected periods ranging between 5.1 and 28.0 months. One of those periods is approximately 7.6 month, which is somewhat similar to a periodicity of 220.0 days found in the present study.

The analysis made in sunspot areas by Lean (1990) during cycles 12-21 showed that the Rieger-type periodicities present only intermittently in each cycle during epochs of maximum activity, and its exact period varies from 130 to 185 days. Richardson \& Cane (2005) studied this periodicity in solar and interplanetary activity levels during solar cycle 23 and reported that, this period occurs intermittently, and also shows a variation in the length of period. However, some questions relating to the origin of these periodicities and no repetition in all of the solar cycle have not been answered until now. As Ballester et al. (2002, 2004) have suggested, the careful and accurate studies of the photospheric magnetic flux may clarify this complexity.

In the present study, the intermediate-term periodicities in the daily sunspot numbers and flare index data, for solar 

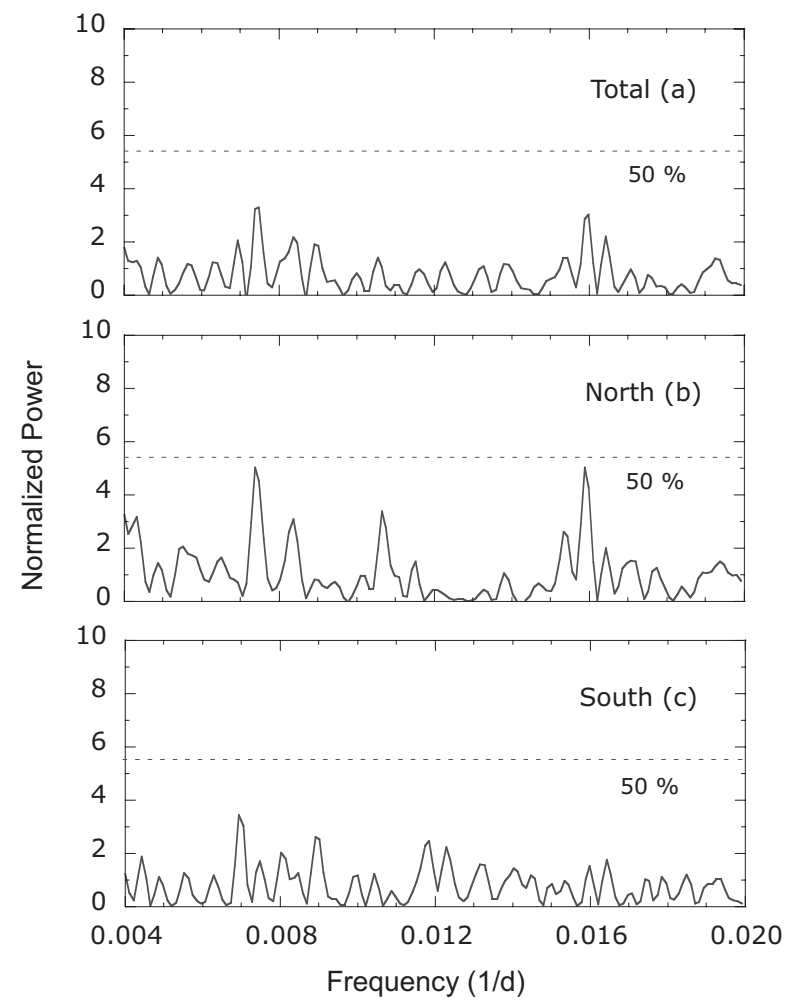

Fig. 4. Same as Fig. 2, but for daily flare index.

cycle 23 from August 23, 1997 to December 31, 2005 were analyzed using the DCDFT. The periodicities in sunspot numbers are generally found to be in agreement with results obtained in previous studies. However, the study shows that the discrepancies in the periodic variations in the northern and southern hemispheres of the Sun display a kind of asymmetrical behavior. On the other hand, the flare index data covering the same time interval does not exhibit any significant periodicity. We conclude that this study illustrates the utility of the DCDFT technique for the study of solar time series.
Acknowledgements. This work was supported by the Scientific Research Project Unit of Akdeniz University. The author sincerely thanks the referee for his/her constructive suggestions and positive comments, and is grateful to the SIDC for the sunspot data, and the NGDC and the Kandilli Observatory for flare index data. Analysis was performed using the computer program TS, developed by the American Association of Variable Star Observers. Beside, the author thanks to Prof. Dr. Zeki Aslan from the TUBITAK National Observatory for his useful suggestions.

\section{References}

Ataç, T., \& Özgüç, A. 2006, Sol. phys., 233, 139

Bai, T. 1987, ApJ, 318, L85

Bai, T. 1992, ApJ, 388, L69

Bai, T. 2003, ApJ, 591, 406

Bai, T., \& Cliver, E. W. 1990, ApJ, 263, 299

Bai, T., \& Sturrock, P. A. 1991, Nature, 350, 141

Bogart, R. S., \& Bai, T. 1985, ApJ, 299, L51

Ballester, J. L., Oliver, R., \& Baudin, F. 1999, ApJ, 522, L153

Ballester, J. L., Oliver, R., \& Carbonell, M. 2002, ApJ, 566, 505

Ballester, J. L., Oliver, R., \& Carbonell, M. 2004, ApJ, 615, L173

Carbonell, M., \& Ballester, J. L. 1990, A\&A, 238, 377

Carbonell, M., \& Ballester, J. L. 1992, A\&A, 255, 350

de Jarger, O. C. 1987, Ph.D. Thesis, Potchefstroom University, Potchefstroom

Delache, Ph., Laclare, F., \& Sadsoud, H. 1985, Nature, 317, 416

Ferraz-Mello, S. 1981, AJ, 86, 619

Foster, G. 1995, AJ, 109, 1889

Horne, J. H., \& Baliunas, S. L. 1986, ApJ, 302, 757

Joshi, B., \& Joshi, A. 2005, Sol. Phys., 226, 153

Joshi, B., Pant, P., \& Manoharan, P. K. 2006, A\&A, 452, 647

Kane, R. P. 2005, Sol. Phys., 227, 155

Kile, J. N., \& Cliver, E. W. 1991, ApJ, 370, 442

Krivova, N. A., \& Solanki, S. K. 2002, A\&A, 394, 701

Landscheidt, T. 1986, Sol. Phys., 107, 195

Lean, J. L. 1990, ApJ, 363, 718

Lean, J. L., \& Brueckner, G. E. 1989, ApJ, 337, 568

Oliver, R., \& Ballester, J. L. 1995, Sol. Phys, 156, 145

Oliver, R., Ballester, J. L., \& Baudin, F. 1998, Nature, 394, 552

Özgüç, A., \& Ataç, T. 1989, Sol. Phys., 123, 357

Özgüç, A., \& Ataç, T. 1994, Sol. Phys., 150, 339

Richardson, I. G., \& Cane, H. V. 2005, Geophys. Res. Lett., 32, L02104

Rieger, E., Share, G. H., Forrest, D. J., et al. 1984, Nature, 312, 623

Scargle, J. D. 1982, ApJ, 263, 835

Templeton, M. 2004, JAAVSO, 32, 41 